LBL--1999 1

DE86 005276

AN ANALYSIS OF SELF-AMPLIFIED SPONTANEOUS EMISSION

$$
\text { Kwang-Je Kim }
$$

Center for X-ray Optics

Lawrence Berkeley Laboratory

University of California

Berkeley, California

November 1985

Invited talk presented at the

Seventh International Free Electron Laser Conference

8-13 September 1985

Granlibakken, California

This work was supported by the U.S. Department of Energy under Comtract NO. DE-RCO3-776SFO0098. 


\title{
AN ANALYSIS OF SELF-AMPLIFIED SPONTANEOUS EMISSION
}

\author{
Kwang-Je Kim \\ Center for X-ray Optics \\ Lawrence Berkeley Laboratory \\ University of California \\ Berkeley, California 94720
}

November 1995

\section{Abstract}

The following analysis develops a classical theory of how a signal evolves from the initial incoherent spontaneous emission in long und.lators. The theory is based on the coupled Klimontovich-Maxwell equations. Formulas for the radiated power, spectral characteristics and electron correlations are derived. The saturation due to nonlinear effects is studied using a quasi-linear extension of the theory. The results agree reasonably well with the recent Livermore experiment in the microwave range. Performance of a possible high-gain free electron laser in a short-wavelength region is evaluated.

1. Introauction

As an electron beani passes through an undulator, the initial random field of spontaneous radiation becomes amplified in intensity and enhanced in coherence characteristics. This process can be called self-anp! if ied spontaneous emission (SASE), and it arises because the interaction between the radiation field and the beam causes a bunching in the beam. An understanding of SASE is important in characterizing the performance of a ligh-ga in free plectron laser, operating in a single-pass mode to circumvent the need for mirrors, in the shortwavelength region [1]. 
To analyze SASE, it is necessary to generalize the usual free electron laser (FEL) analysis in two respects. Firsî, a continuum of the frequency range around the resonant frequency must be explored since the spectrum characteristics change as the system evolves. Second, the discreteness of the electron distribution must be taken into account, since otherwise spontaneous emission is not possible. This is accomplished in this paper by working with the $\mathrm{Klimontovich}$ distribution function [2], rather than Vlasov's. The coupled Klimontovich-Maxwell equations are solved by perturbation theory, in which deviations of the fine-grained distribution from the smooth average is regarded as being a first-order quantity. One finds that the radiation field is composed of two terms. The first term is proportional to the input coherent signal and describes the well-known FEL gain process. The second term is proportional to the sum of random phase factors and represents the SASE process.

The radiation intensity corresponding to the SASE term reduces to the well-known result for spontaneous emission in the limit of small interaction. In the regime of exponential growth, one obtains an explicit formula for the power and the spectral characteristics of the SASE radiation, as well as insights into the correlation properties of the electron beam distribution. The exponential growth saturates eventually due to nonlinear effects, which can be analyzed in a quasilinear approximation [3]. These results are then used to discuss the recent microwave FEL experiment at Livermore [4] and to assess the performance of a high-gain, single-pass FEL im the short-wavelength region [5]. 
II. The Klimontovich-Maxwell Equations

The average energy of the electron beam will be denotid by $\mathrm{mc}^{2} \boldsymbol{r}_{0}$ ( $m=$ electron mass, $c=$ velocity of light). The beam travels along the z-direction through an undulator of period length $\lambda_{u}$ with a peak magnetic field $B_{0}$. The resonant frequency $\omega_{1}$ and the corresponding wavelength $\lambda_{1}$ are given by

$$
\omega_{1}=\frac{2 \pi c}{\lambda_{1}}=k_{u} c \frac{2 \gamma^{2}}{1+k^{2} / 2},
$$

where $\mathrm{K}=\mathrm{e} \mathrm{B}_{\mathrm{o}} / \mathrm{mck}_{u}$, e = electron charge and $k_{u}=2 \pi / \lambda_{u}$. (MKS units are used throughout this paper.) for a one-dimensional case, the electric field can be represented by

$$
E(z, t)=\frac{\omega_{1}}{\sqrt{2 \pi}} \int_{-\infty}^{\infty} d v A^{\prime}(v, z) e^{i v \omega_{1}(t-z / c)}
$$

$A^{\prime}$ in Eq. (2) is a complex amplitude slowly varying in $z$ and is peaked around $|\nu| \sim 1$. Although the theory can be readily generalized to higher harmonic, the focus in this paper will be the fundamental frequency. The distance $z$ from the undulator entrance will be chosen as the independent variable. The dependent variables describing the motion of the i-th electron are

$$
e_{i}=k_{u} 2-w_{1}\left(\bar{t}_{i}(2)-2 / c\right)
$$




$$
n_{i}=\frac{r_{i}-r_{0}}{r_{0}}
$$

Here $t_{j}(z)$ is the time at which the electron passes through $z$, averaged over the wiggling motion. The equations of motion, usually known as the pendulum equations, are as follows:

$$
\begin{aligned}
& \frac{d \theta_{i}}{d z}=2 k_{u^{n}} n_{i}, \\
& \frac{d n_{i}}{d z}=\frac{e k[J J]}{2 \gamma_{0}^{2} m c^{2}} \tilde{A}\left(\theta_{i}\right)
\end{aligned}
$$

Here

$$
\begin{aligned}
& {[J]=\left[J_{0}(\xi)-J_{1}(\xi)\right], \xi=\frac{k^{2}}{4\left(1+k^{2} / 2\right)},} \\
& \tilde{A}(\theta)=\frac{1}{i_{\pi} \cdot \pi} \int i v A(v) e^{-i v \theta}, \\
& A(v)=A^{\prime}(v) e^{-i \Delta v k} u^{2}, \\
& \Delta v=v \cdot 1 .
\end{aligned}
$$

The $K l$ inmontovich distribution function is

$$
\begin{aligned}
\tilde{F}(\theta, n, z) & =\frac{2 \pi}{N_{\lambda}} \sum_{j} s\left(\theta-\theta_{j}(z) s\left(n-m_{j}(z)\right)\right. \\
& =V(n)+\delta \tilde{F}(\theta, m, z) .
\end{aligned}
$$


Here $N_{\lambda}$ is the number of electrons within a longitudinal distance equal to $\lambda_{1}$ and $V(n)$ is the smoothed initial distribution function (normalized so that $\int v d_{n}=1$ ), the distribution in $\theta$ being assumed uniform. $\delta \tilde{F}$ in Eq. (11) contains the deviation from the smooth background, as well as the effects of the interaction, and will be treated as a small, first-order quantity. The continuity equation in $\theta-n$ space becomes

$$
\left(\frac{\partial}{\partial z}+2 k_{u} \eta \frac{\partial}{\partial \theta}\right) \delta \tilde{F}+\frac{e K[J J]}{2 r_{0}^{2} m c^{2}} \tilde{A}(\theta) \frac{\partial}{\partial \eta} V(\pi)=0
$$

In equation (12), a term containing the product $A(\theta) \delta \tilde{F}$ has been dropped as being a second-order term. Later, the term will be retained to study the saturation effects in a quasi-linear theory.

The Maxwell equation is

$$
\left(\frac{\partial}{\partial z}-i \Delta v k_{u}\right) A(v, z)=-\frac{K[J J] j}{4_{r_{0} \omega^{\omega}}} \int \delta F(v, n) d n,
$$

where $j$ is the current density, $z_{0}=377$ Ohms and

$$
\delta F(v, n)=\frac{1}{\sqrt{2 \pi}} \int e^{i v \theta} \delta \tilde{F}(\theta, n) d \theta
$$

\section{The Solutions}

The coupled Klimontovich-Maxwe 11 equations, Eqs. (12) and (13), are identical in structure to the usuad vlasov-Maxwell equations and can be solved in a inear theory with the Laplace-transfonm technique. One obtains 


$$
A(v, z)=\oint \frac{d x}{2 \pi i} \frac{e^{-2 i k} u^{2 x}}{D(x, v)}\left(A(v, 0)+d \int d_{\eta} \frac{\delta F(v, n, 0)}{x+n v}\right),
$$

where $d=K[J J] Z_{0} j / 8 i \gamma_{0} \omega_{1} k_{u}$. The contour integration in Eq. (15) effects the inverse Laplace transformation and should enclose the appropriate poles in the integrand. In addition to poles of kinematic origin, the poles obtained by solving the dispersion relation

$$
D(x, v)=x+\frac{\Delta \nu}{2}+\rho 3 d \pi \frac{d V / d n}{x+\pi \nu}=0,
$$

determine the dynamics of the system. Here $n$ is a dimensionless parameter characterizing the interaction strength [6] and is given by

$$
\omega=\left(\frac{e K[J j] z_{0} j}{32 \gamma_{0}^{2} m c^{2} k_{u}^{2}}\right)^{1 / 3}
$$

Equation (16) is essentially the cubic equation known in the literature [7], but generalized to include the effect of the beam energy spread and the detuning in frequency.

Equation (15) gives the solution in terms of the initial condition $A(v, 0)$ and $\delta F(v, n, 0)$. The term proportional to the former describes the amplification of the coherent input signal and reproduces the well-known theory of FEL interaction [8]. Since the main purpose of this paper is to study the SASE process, the first term will not be considered further. The second term, the SASE term, contains a sum of stochastic phase factors which vamishes iff averaged over macroscopically equivalient ensenthes. However, physically meaningfun quamtities

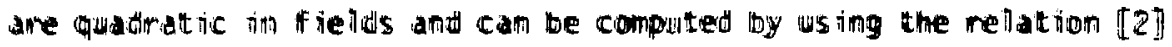




$$
\left\langle\delta \tilde{F}(\theta, n, 0) \delta \tilde{F}\left(\theta^{\prime}, n^{\prime}, 0\right)\right\rangle=\frac{2 \pi}{N_{\lambda}} \delta\left(\theta-\theta^{\prime}\right) \delta\left(n-n^{\prime}\right) V(n),
$$

where the angular brackets denote the ensemble average.

The spectral distribution of power is given by

$$
\frac{d P}{d \omega}=\frac{2 C o}{Z_{0} l}\left\langle A(v, z) A^{\star}(v, z)\right\rangle,
$$

where $\sigma$ is the beam cross-sectional area and $\ell$ is the bunch length. IV. Spontaneous Radiation

By dropping the last term in the dispersion relation (Eq. (16)), Eq. (15) and the power spectrum given by Eq. (19) are easy to evaluate. To compare the result of evaluating Eq. (19) with a known formuia one Ilultiplies $\mathrm{dP} / \mathrm{d} \omega$ by $\delta^{2}(\phi)$ to obtain the angular distribution. In the forward direction, the factor becones $\delta^{2}(0)=\sigma / \lambda_{1}^{2}$. One obtains

$$
\left.\frac{d P}{d \omega d^{2} \phi}\right|_{\phi=0}=\frac{z_{0}}{16 \pi^{3}}\left(\frac{k[J J]}{1+k^{2} / 2}\right)^{2} \gamma^{2} \frac{I}{e} \int \operatorname{dnV}(n)\left(\frac{\sin k_{u} z(n v-\Delta v / 2)}{n v-\Delta v / 2}\right)^{?},
$$

where I is the beam current $a j$. Equation (20) is well-known in the theory of undulator radiation [9].

V. SASE in the Exponential Gain Regime

In general, the dispersion relation has a solution $x=n u$, with a positive imaginary part $x_{1}$ that gives rise to an exponentia!ly gruwing intensity term proportionall to exp (4k $\left.k_{y} \tau^{2} z\right)$. The spectrall property is determimed by the behawior of $x_{\mathbb{1}}$ as fumction of detuning av. For 
a given momentum distribution $V(n)$, let $x_{I}{ }^{m}$ be the maximum value of $x_{I}$ at $\Delta v=\Delta v_{m}$. Thus the growth is strongest at frequency $\omega=\omega_{m}=$ ${ }^{\omega} 1\left(1+\Delta v_{m}\right)$, ano the spectral shape is obtained by studying the behavior of $x_{I}$ near $\Delta v=\Delta v_{m}$. One obtains

$$
\frac{d P}{d \omega}=\rho \frac{m c^{2} \gamma_{0}}{2 \pi} g e^{\tau} e^{-\frac{\left(\omega-\omega_{m}\right)^{2}}{2\left(\omega_{1} \sigma_{v}\right)^{2}}},
$$

where

$$
\begin{aligned}
& \tau=4 k_{u} x_{I}^{m} z, \\
& g=\frac{\operatorname{sdn} V(n) /\left.\right|_{\mu}+n /\left.\rho\right|^{2}}{|d D / d x|_{x}^{2}=\rho \mu} .
\end{aligned}
$$

In Eq. (21) $\sigma_{v}$ is the rms value of the relative bandwidth. For the ideal case, where $V(n)=\delta(n)$, one obtains [10]

$$
g=\frac{1}{9}, \quad x_{I}^{m}=\rho \frac{\sqrt{3}}{2} \quad \text { and } \quad v_{v}=\sqrt{\frac{9 \rho}{2 \pi \sqrt{3}\left(z / \lambda_{u}\right)}} \text {. }
$$

The exponential growth of SASE saturates when $p 2 / \lambda_{u}$ becomes of order unity, as will be seen later. The bandwidth of SASE in the exponentially growing region is smaller by a factor $\left(02 / x_{u}\right)^{1 / 2}$ than tllat of the spontaneous radiation, which is about $x_{u} / 2$. However, the bandwidths of SASE and the spontaneovis radiation are comparable at saturation.

For more general V(t), the dispersitom rellation must the solved mumericilliy to obtaim $x_{1}$, etic. The resuntis for a rectangullar 
distribution are summarized in Fig. (1) and Fig. (2). One notices that the growth rate becomes negligible when the width of $V(n)$ is much larger than $n$.

The total power is obtained by integration, whereby one obtains

$$
P_{T}=\int \frac{d P}{d \omega} d_{\omega}=\rho P_{\text {beam }} \frac{\sqrt{2 \pi} \sigma_{\nu}}{N_{\lambda}} g e^{\tau} .
$$

Here $P_{\text {beam }}=m c^{2} r_{0} I / e$ is the power contained in the electron beam. VI. Correlation

By studying the solution for $\delta \tilde{F}(\theta, n, z)$, one obtains the correlation function in the exponential growth region

$$
\begin{aligned}
C\left(\theta, \theta^{\prime}, z\right) & =\iint d \eta d \eta^{\prime}\left\langle\delta \tilde{F}(\theta, n, z) \delta_{i}\left(\theta^{\prime}, \eta^{\prime}, z\right)\right\rangle \\
& =\frac{2 \sqrt{2 \pi} \sigma_{v}}{9 N_{\lambda}} e^{\tau} e^{-c_{\nu}^{2}\left(\theta-\theta^{\prime}\right)^{2} / 2} \cos \left(\theta-\theta^{\prime}\right) .
\end{aligned}
$$

One sees that the correlation, modulated with the periodicity of the radiation wavelength, decreases as the distance between the electrons increases.

\section{Saturation and Quas $i-l$ inear Theory}

The exponential growth cannot continue indefinitely, and the power must saturate at a certain level. The effect is due to nonl inear effects and can be studied by a quasi-linear extension of the linear theory [3]. For this purpose, one replaces V(m) in Eq- (11) by a $z$-dependemt function $V(m, z)$ which is obtained from $\langle\vec{F}\rangle$ by averaging over as follows: 


$$
V(n, z)=\frac{{ }^{\lambda} 1}{2 \pi l} \int d \theta \cdot \tilde{F}(\theta, n, z)>
$$

The continuity equation for $V(n, z)$ is

$$
\frac{\partial V}{\partial z}+\frac{e K}{2 Y_{0}^{2} m c l} \frac{\partial}{\partial \eta} \int d v\left(A_{v} \delta F_{v}{ }^{*}+c . c .\right)=0 \text {. }
$$

In a quasi-linear theory, one solves the linear equations (12) and (13), treating $V$ as $z$-independent, and obtains $A_{v}$ and $\delta F_{v}$ as functionals of $V$. Inserting these into Eq. (28), one obtains a nonlinear Fokker-Planck equation which determines the behavior of $V$ as a function of $z$. In this way it is found that the average value of $n$ decreases so as to conserve the total energy of the radiation-electron beam system. It is also found that the rms spread $a_{\eta}$ of $\eta$ increases as [11]

$$
i_{n}^{2}-\rho^{2}\left(\frac{\sqrt{2 \pi} \sigma_{u}}{9 N_{\lambda}} e^{\tau}\right)
$$

On the other hand, the growth $r$ ate becomes negligible when $\sigma_{\eta} \gg 0$. Thus, SASE saturates when the factor in the bracket in Eq. (29) becomes of order unity. In view of Eq. (25), the saturated power is

$$
P_{\text {sat }}-P_{\text {beam }} \text {. }
$$

This relation was derived before using an intuitive argument [6]. VII. Comparisot with the livermore Exper iment

A migh-gain FEL experinnerit the microwave region has been carried out at Livenmore [4]. Using the parameters of the experinent $\left(\pi_{0}=7\right.$,

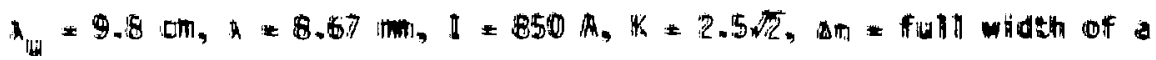


rectangular momentum distribution $=6.4 \%, \sigma=3 \times 10 / 2 \mathrm{~cm}^{2}$ ), one obtains $\rho=5.66 \times 10^{-2}$ and the corresponding growth rate or $42.1 \mathrm{~dB} / \mathrm{m}$. The observed growth rate is $35 \mathrm{~dB} / \mathrm{m}$ which is smaller than that predicted because of space charge effects. Taking the observed growth rate and computing the coefficient $i=5 q .(25)$, one finds

$$
\mathrm{P}_{T}(\text { Watts })=2.8 \times 10^{-5} \times 10^{3.5 z(\mathrm{~m})} / \sqrt{2(\mathrm{~m})} .
$$

Figure (3) compares this formula with the experimental result. For sinall $z$, the theoretical values are less than the experimenta? points, which could be due to the presence of other modes, e.g., highe: harmonics. For $z \geq 2 m$ the theoretical value is higher, which could be due to the saturation effects.

VIII. A High-Gain FEL at $40 \mathrm{C} \AA$

A high-gain FEL operating in a special by-pass of an optimized storage ring is a proinising way to achieve high-power radiation at short wavelengths [1]. A design of such a system for a $400 \AA$ FEL is Jescribed in Ref. [5], where $\circ-1.5 \times 10^{-3}$ for an electron beam with I - $200 \mathrm{~A}$ and an rms nomentum sprerd of $2 \%$, which translates to a full width of $7 \%$ for a rectanguldr distribution. Such a system would generate about $100 \mathrm{Mw}$ of peak power, saturated at around 1000 undulator perioos. The rellative band width of the spectrum would be around $10^{-3}$. 1.. Conclusion

The tineory prescmlted there is a coms istent, classical treatment of ithe development of a cotherent stignal from imitial maise [?,?]. The

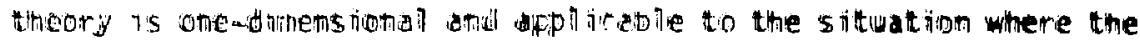


radiation is guided, such as the Livermore experiment. In general, however, the three-dimensional aspects, suct, as diffraction and finite-beam-size effects, could play an important role $[13,14]$. These and other extensions of the theory are currently under investigation. Acknowledgments

it is a pleasure to acknowledge some of my colleagues, whose expertise was crucial in the development of +' = ti eory presented here. C.. Pellegrini introduced me to the subject ot higr-gain FELs and the relevant issues. S. Krinsky and J.M. Wang shared their insights on the subject, and E.T. Scharlemiann and T.J. Orzechowski have been very generous in taking their time to explain the Livermore experiment.

This work was supporied by the U.S. Department of Energy under Contract. No. DE-AC03-76SF00098. 


\section{References}

[1] J. Murphy and C. Pellegrini, J. Opt. Soc. Am. 1B (1984) 530.

[2] Y.L. K.1 imontovich, Soviet Phyr. JETP 6 (1958) 753. See aiso S. Ichimaru, Basic Principles of Plasma Physirs (W.A. Benjamin, Inc., 1973) Appendix A.

[3] See, for example, G. Schmidt, Physics of High Temperature Plasmas (Academic Press, New York, 1979) Section 9-2.

[4] T.J. Orzechowski et al., High Gain and High Extraction Efficiency from a Free Electron Laser Amplifier Operating in the Millineter Wave Regime, these proceedings; Phys. Rev. Lett. 54 (1985) 889 .

[5] J.J. Bisognano et al., Feasibility Study of a Storage king for a High Power XUV Free Electron Laser, preprint LBL-19771, submitted to Particle Acc.; K-J. Kim, J.J. Bisognano, A.A. Garren, $\ddot{i}$. rialbach and J.M. Peterson, Nucl. Inst. and Methods A239 (1985) 54 .

[6] B. Bonifacio, C. Pellegrini and N. Narducci, in Free Electron Generation of Extreme UItraviolet Coherent Radiation, J.M.J. Madey and C. Pellegrini, eds. (Am. Inst. Phys., New York, 1984) 236.

[7] N.M. Kroll and W.A. McMullin, Phys. Rev. Al7 (1978) 300; A. Gover and 2. Livni, Opt. Comm. 26 (1978) 375.

[8] See for example, H.B. Colson, Phys. Lett. 64A (1977) 190.

[9] See for example, S. Krinsky, JEEE Trans. Hucl. Sci. NS-30 (1983) 3078. 
[10] This result has been also obtained by J.M. Wang and L.H. Yu (private comunication) using a Green's function method.

[11] S. Krinsky, Proceedings of 1985 SLAC Summer Schools on High Energy Particle Accelerators, Stanford Lincar Accelerator Center (to be published).

[12] For previous analysis on the related subject, see P. Sprangle, C.M. Tang and L. Bernstein, Phys. Rev. Lett. 50 (1983) 1775, A.T. Georges, Free-E\}ectron Generation of Coherent Radiation, SPIE Proc. 453 (1983) 297.

[13] G.T. Moore, Optics Comm. 54 (1985) 121.

[14] E.T. Scharlemann, A.M. Sessler and I.S. Wurtele, Phys. Rev. Lett. 54 (1985) 1925. 


\section{Figure Captions}

Fig. 1. The solution of the dispersion relation for arbitrary harmonic number $n$ ( $n=1$ in the rest of this paper). The curves show the values of $x_{1} / \rho n^{i / 3}$ as functions of $\Delta w /\left(\omega_{1} \rho n^{1 / 3}\right)$ for various values of $\delta=\delta n n^{2 / 3} / n$. The momentum aistribution is assumed to be rectangular; $V(n)=$ $1 / \Delta n$ for $|n| \leq \Delta n / 2$ and $V(n)=0$ for $|n| \geq \Delta n / 2$.

Fig. 2. The behavior of $\operatorname{Max}\left(\mu_{I}\right)=X_{I}^{m}$ is and $g(E q .(23))$ as functions of $\Delta \eta / \rho$.

Fig. 3. A comparison of the experimental results (solid dots) and the theoretical prediction (dotted line) corresponding to the Livermore experiment (Ref. [4]). 


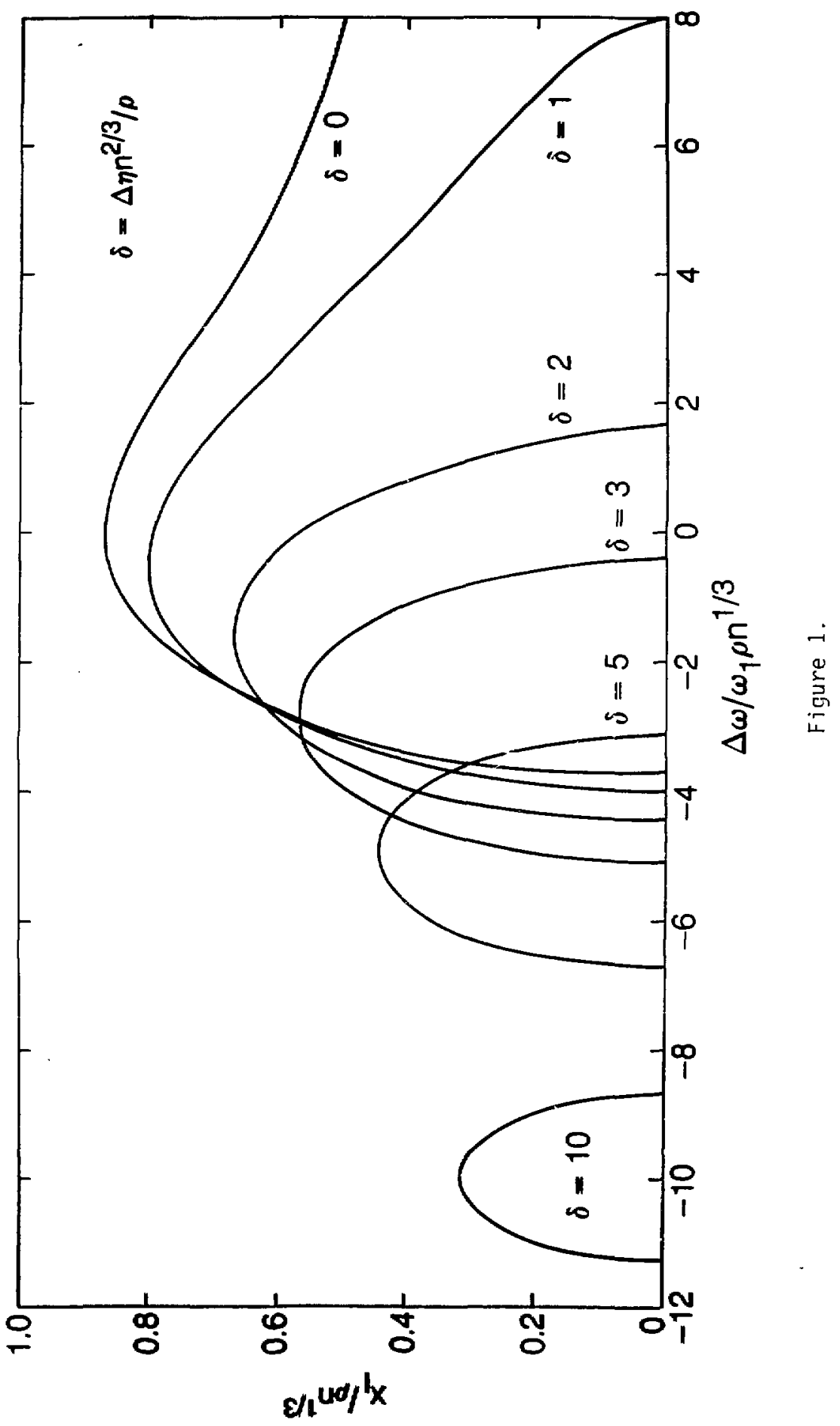




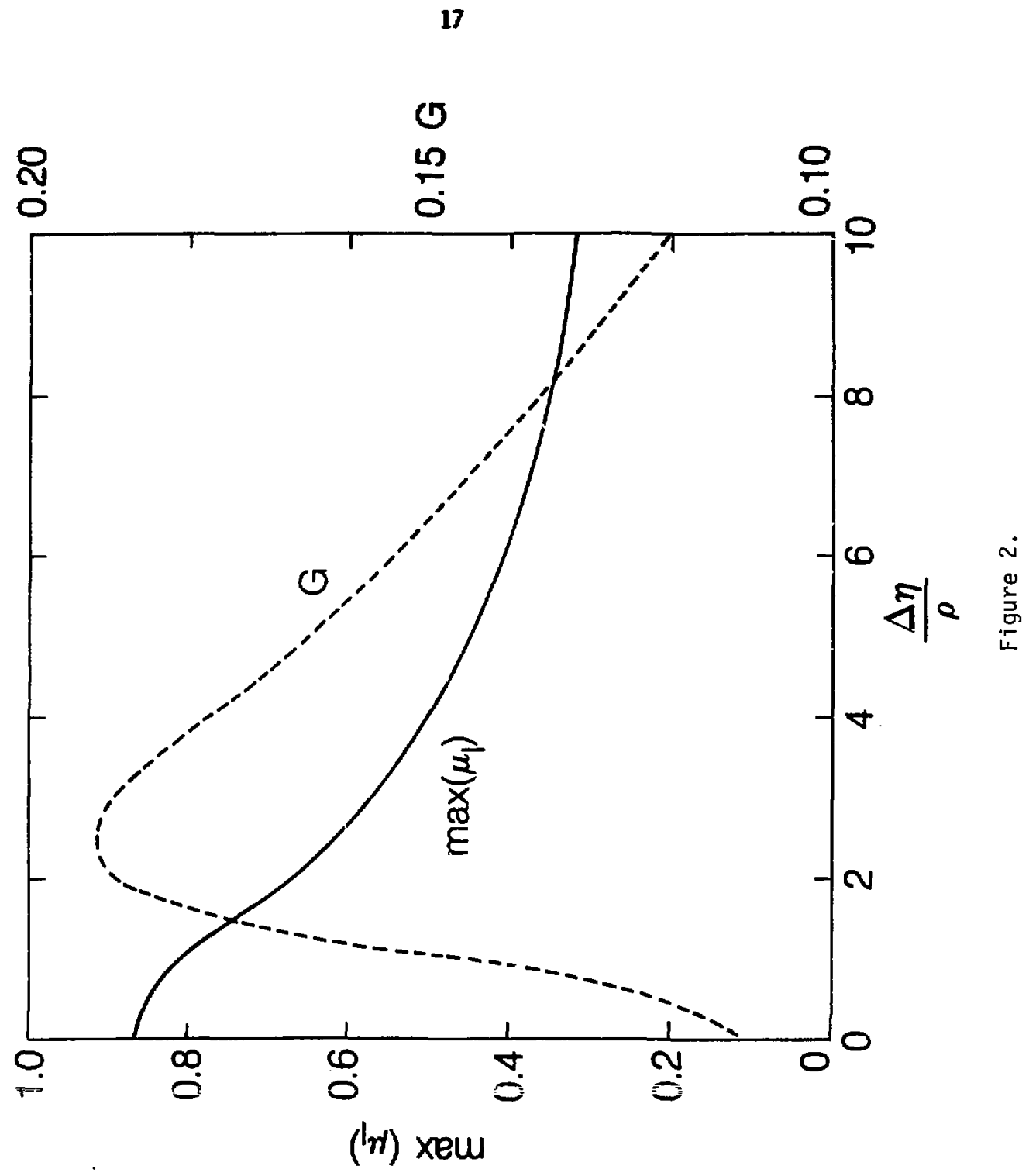




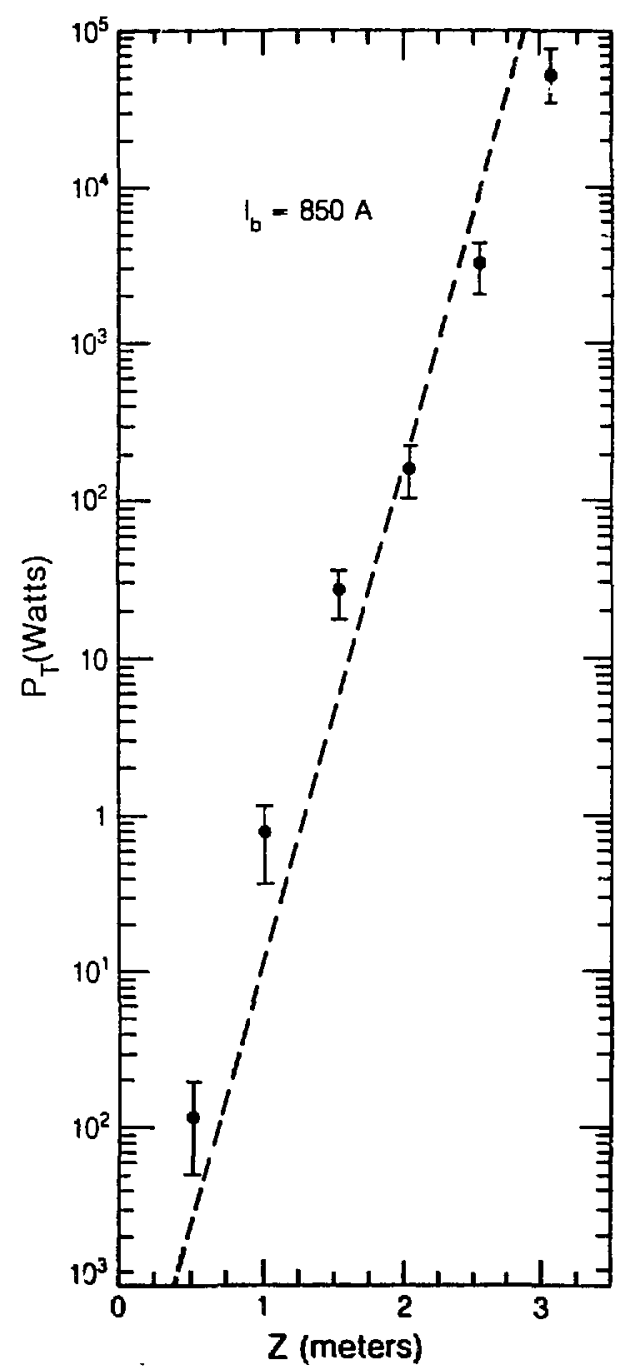

Figure 3. 
This report was done with support from the Department of Energy. Any conctusions or opinions expreseed in this report represent wolely those of the author(s) and not necessurily those of The Refitnts of the University of Californin, the Lawnence bisidely Laboratory or the Departinent of Enerty.

Reference to e company or product mine does not imply approval or recommendation of the product by the Univerity of Califormin or the U.S. Department of Energy to the exclusion of others that may be suitable. 